\title{
La etapa de contacto líquido-líquido operando en corriente cruzada ${ }^{(\bullet)}$
}

\author{
J.L. Otero de la Gándara ${ }^{(*)}$
}

\begin{abstract}
Resumen Se describe una nueva variante de etapa de contacto líquido-líquido. El flujo de fases se realiza fraccionando la fase orgánica en varias corrientes y la fase acuosa se desplaza a través de conductos ascendentes y descendentes dispuestos en zig-zag y conectados en serie. Cada fracción de flujo de fase orgánica se alimenta en la base de los conductos en los que la fase acuosa asciende. En estos conductos tiene lugar la transferencia de materia entre fases, que se facilita mediante la utilización de un relleno convencional. La coalescencia de la fase orgánica se produce en la zona superior de estos conductos. La etapa se comporta produciendo una variación de concentraciones superior a la correspondiente a una etapa teórica convencional. Se resalta la reducción importante del consumo de energía en el contacto de fases y el bajo retenido de fase orgánica, en relación con un mezcladorsedimentador convencional.
\end{abstract}

Palabras clave: Etapa. Extracción líquido-líquido. Corriente cruzada.

\section{The liquid-liquid contact stage operating in cross current}

\begin{abstract}
A new type of the liquid-liquid contact stage is described. The phase flow is performed by splitting of the organic phase into several currents with no splitting aqueous phase. The water phase is driven through ascending and descending conducts arranged in zig-zag paths and conected in serie. Each splitted flow of organic phase is fed at the bottom of the conducts where the aqueous phase rises. The transfer of the matter between phases take place within these conducts improved by means of a conventional packing. The coalescence of the organic phase occurs at the top of the conducts. The stage yields a concentration change larger that a theoretical conventional stage. The new type of contact stage points out an important reduction of power and a low hold-up of organic phase when is compared to a conventional mixer-settler.
\end{abstract}

Keywords: Stage. Liquid-liquid extraction. Cross current.

\section{INTRODUCCIÓN}

Cuando se utiliza la operación de extracción líquido-líquido en hidrometalurgia, se emplea como sistema mecánico de transferencia entre fases, en un elevado número de ocasiones, etapas de mezcladores-sedimentadores. Con estos sistemas se consigue: a) un buen contacto entre fases y, en consecuencia, una eficacia elevada, próxima o igual de $100 \%$ del equilibrio fisicoquímico, b) operar en un amplio intervalo de caudales, c) costes de instalación y mantenimiento bajos, y d) un fácil cambio de escala, al pasar de resultados de laboratorio o planta

$(\bullet)$ Trabajo recibido el día 10 de abril de 1995.

(*) Dpto. de Ingeniería Química. Facultad de Ciencias Químicas. Universidad Complutense de Madrid. 28040Madrid (España). piloto a escala industrial. Junto a estas ventajas se tienen como inconvenientes: a) un retenido de fase acuosa y orgánica elevado y, dado el coste de esta última, un importante valor del inmovilizado en fase orgánica, b) un consumo elevado de energía en mezcla de fases, y c) en algunos casos, una importante superficie ocupada por la instalación.

Dados los inconvenientes resaltados, es lógico que se haya intentado modificar los diseños mecánicos, reduciendo el retenido de fases y los costes de energía en mezcla y bombeo, así como hacer posible que el equipo se pueda instalar a la intemperie, con la consiguiente reducción de la inversión en naves.

La mezcla de fases se puede efectuar en cámaras simples o múltiples conectadas en serie. La utilización de una sola cámara es la solución adoptada con mayor frecuencia. La separación de fases tiene lugar en la misma cámara de mezcla o en una 
cámara contigua. Se distingue en el recinto de separación una zona de dispersión de ambas fases y otras dos zonas, una superior de fase orgánica y otra inferior de fase acuosa.

En los equipos Davy Power Gas y Denver, la mezcla de fases pasa por rebose de la cámara de mezcla a la de separación contigua. En la etapa General Mill, la mezcla se efectúa en un depósito cilíndrico y el rebose fluye a la cámara de decantación a través de una zona con placas enderezadoras de corriente, con el fin de reducir el tiempo necesario para la separación de fases. En el modelo del Imi, el rebose de fases mezcladas se hace llegar a la zona central de un depósito cilíndrico contiguo. Este conducto de llegada está rodeado de un sistema de placas antiturbulencias y directoras de flujo. El dispositivo Kemira puede considerarse parecido al Imi, desprovisto de las placas. El modelo Lurgi, emplea un sistema múltiple de amplias placas horizontales para fraccionar la separación de fases en cámaras parcialmente independientes. El modelo convencional Davy Power-Gas descrito, se ha modificado situando el mezclador rodeado de cámaras múltiples de sedimentación, dispuestas en corona, provistas de placas para favorecer la separación de fases. La conexión del mezclador con cada cámara, a través de un conducto con válvula, permite su limpieza individualizada sin exigir la parada de la instalación.

En la etapa Holmes-Narver, a continuación del mezclador, se sitúa un canal de sedimentación en el que para facilitar la separación, las fases fluyen guiadas por placas verticales, que las dirigen en forma alternada de la superficie al fondo y del fondo a la superficie.

En (1) se presenta una revisión de los diferentes sistemas descritos; en (2-6), las referencias a constructores de equipo.

En los sistemas mecánicos de mezclas de fases se intenta no alcanzar un tamaño de gotas de fase orgánica muy pequeñas, que presentan una gran dificultad para coalescer y son arrastradas por la fase acuosa, siendo las causantes principales de las pérdidas de fase orgánica en la operación. Las placas que dirigen el flujo, reducen turbulencia y facilitan la separación de fases, siguen siendo tema de estudio y mejora.

El acoplamiento de etapas se efectúa intentando elimiar la necesidad de instalaciones de bombeo, mediante circulación por gravedad. Los sistemas mecánicos de mezcla suelen diseñarse imponiéndoles también la función de bombeo.

Los diferentes fabricantes de equipos descritos valoran sus ventajas basándose, en general, en afirmaciones semicuantitativas no muy rigurosas y sin la comparación de sus características en base clara, ya que no se han utilizado con sistemas o circunstancias idénticas. No obstante, puede afirmarse que cuando se alcanzan eficacias de contacto altas, utilizando siste- mas que mezclan sin turbulencia elevada, y se emplean placas en la separación de fases, se consiguen las etapas de contacto con las mejores características.

A continuación, se describe un concepto de etapa de contacto de fases, ( 7 y 8) con un consumo mínimo de energía de bombeo y contacto de fases, una separación de fases que requiere un espacio mínimo y una transferencia de materia superior a la correspondiente a una etapa teórica convencional.

\section{ETAPA DE CONTACTO LÍQUIDO- LÍQUIDO CON CIRCULACIÓN DE FASES CRUZADA}

En la figura 1a), se representa en esquema un corte vertical de la etapa. La fase acuosa circula describiendo un zig-zag (conductos con flujo ascendente y descendente) y la fase orgánica fraccionada en un número de corrientes igual a la mitad de canales en zig-zag. El contacto entre fases se efectúa en corrientes paralelas y con flujo ascendente. En la parte superior de estos conductos tiene lugar la separación de la fase orgánica de la acuosa por coalescencia de la primera en una zona continua de fase orgánica. Desde esta zona, la fase orgánica pasa por rebose a una cámara lateral (Fig. 1b)), en la que se efectúa la mezcla de todas las corrientes ascendentes de fase orgánica.

En los conductos en los que fluyen en paralelo las fases acuosa y orgánica, se dispone un relleno convencional, con altura adecuada para que prácticamente ambas fases alcancen las composiciones del equilibrio fisicoquímico del sistema. En la figura 1c), 1d), 1e) y 1f), se representan diferentes cortes de la etapa, convenientemente identificados, para facilitar la comprensión de su funcionamiento.

En la figura 1g), se da una vista lateral del acoplamiento de dos etapas. La circulación de la fase acuosa se consigue por gravedad y su distribución homogénea mediante un vertedero almenado situado a la salida de la etapa y en la entrada de la primera. La circulación de la fase orgánica se efectúa mediante bombeo. La potencia de bombeo es baja, ya que el desnivel que se puede precisar entre etapas, para la circulación por gravedad de la fase acuosa es pequeño, y también puede ser pequeña la pérdida de presión precisa en los conductos de distribución de fase orgánica y en los orificios que la dispersan homogéneamente en la base de los conductos ascendentes con relleno (Figs. 1e) y 1g)).

\section{ALGORITMO DE CÁlCULO}

En una etapa de contacto entre fases, las composiciones de la fase acuosa, $X_{\mathrm{e}}, X_{\mathrm{s}}$, y de la fase 

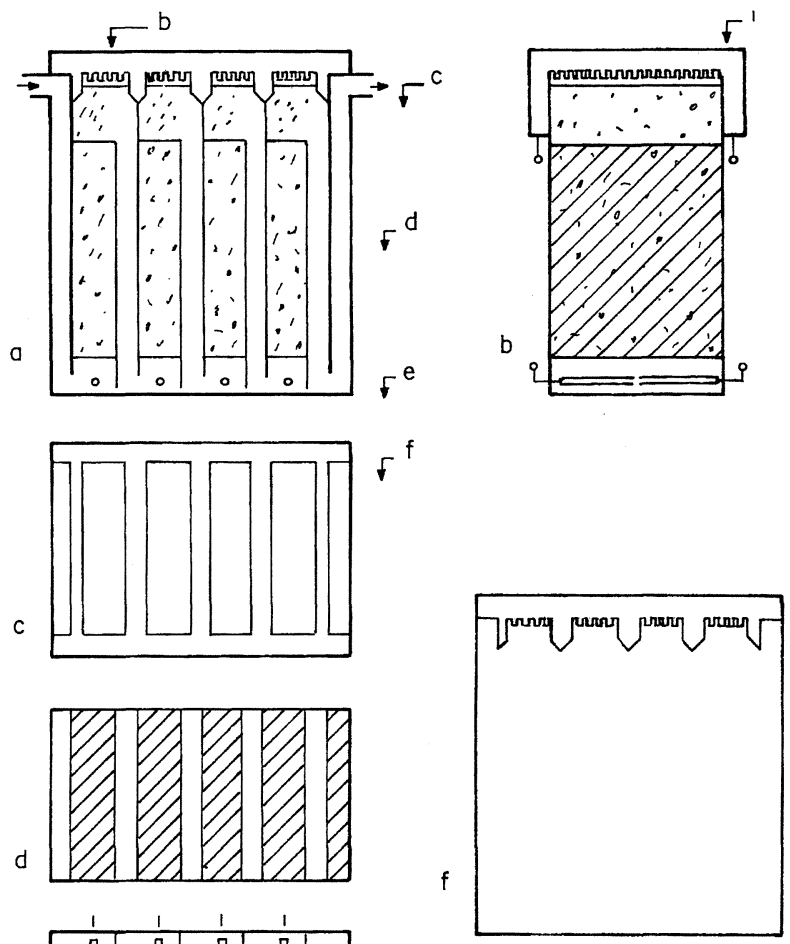

d
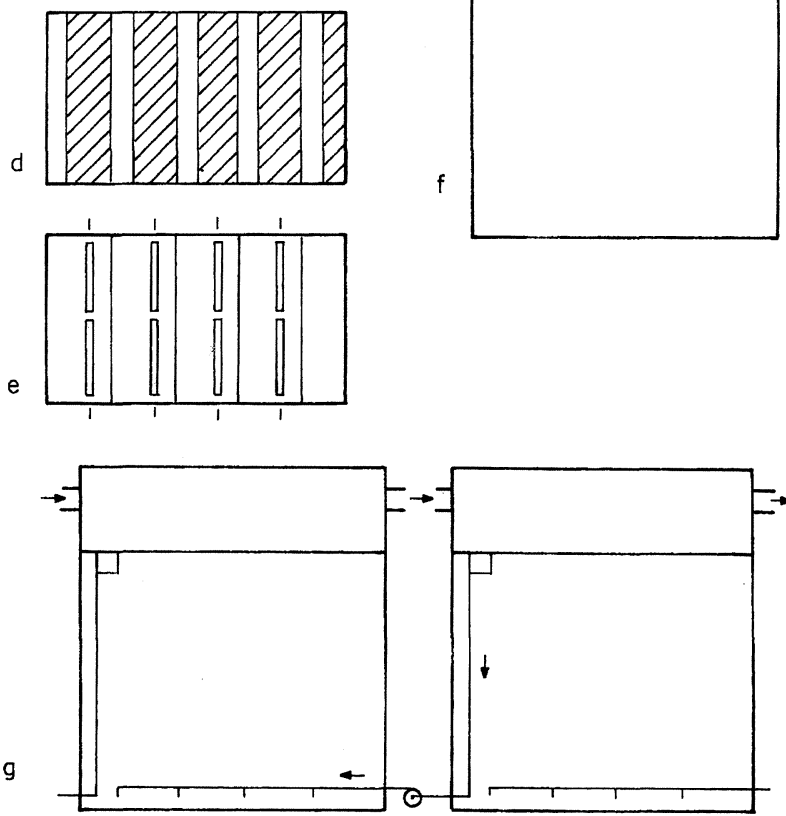

Fig. 1.- Etapa de contacto líquido-líquido, con circulación de fase orgánica segregada en cuatro líneas.

FIG. 1.- Liquid-liquid contact stage with organic phase circulation splitted in four lines.

orgánica, $Y_{\mathrm{e}}, Y_{\mathrm{s}}$, de entrada y salida, respectivamente, operando con caudales $L$ de fase acuosa y $V$ de orgánica, se relacionan mediante la expresión:

$$
X_{\mathrm{e}}=X_{\mathrm{s}}-\frac{Y_{\mathrm{e}}-Y_{\mathrm{s}}}{\frac{L}{V}}
$$

si se alcanza en la etapa el equilibrio fisioquímico, $\left(X_{\mathrm{s}}, Y_{\mathrm{s}}\right)$ cumplen la condición:

$$
Y_{\mathrm{s}}^{*}=f\left(X_{\mathrm{s}}^{*}\right)
$$

Cuando se opera en la etapa, fraccionando la fase orgánica en $N$ líneas en paralelo, en cada uno de los conductos ascendentes se definen las concentraciones de fase mediante:

$$
X_{\mathrm{e}}=X_{\mathrm{s}}-\frac{Y_{\mathrm{e}}-Y_{\mathrm{s}}}{\frac{L}{V} \cdot N}
$$

El valor de $X_{\mathrm{e}}$ en cada conducto ascendente es igual al valor de $X_{\mathrm{s}}$ en el conducto ascendente anterior. En la figura 2 se representa la evolución gráfica de las composiciones, para $N=4, Y_{\mathrm{e}}=0$, en el caso de que en todos los conductos ascendentes se alcancen las composiciones de equilibrio exigidas por (2). La mezcla de los $N$ corrientes de fase orgánica, que abandonan los conductos ascendentes, tiene una composición $Y_{\mathrm{m}, \mathrm{s}}$, que se puede calcular mediante:

$$
Y_{\mathrm{m}, \mathrm{s}}=\frac{\sum_{1}^{\mathrm{N}} Y_{\mathrm{s}}}{N}
$$

Gráficamente en la figura 2, se comprende fácilmente que con $\left(X_{\mathrm{s}} ; Y_{\mathrm{m}, \mathrm{s}}\right)$ se define una función $Y_{\mathrm{m}, \mathrm{s}}$ $=\mathrm{f}\left(X_{\mathrm{s}}\right)$ en la que $Y_{\mathrm{m}, \mathrm{s}}$ es mayor que el valor de $Y_{\mathrm{s}}$ obtenido si se opera con $N=1$.

Dadas las características de los diagramas de equilibrio, tanto en las operaciones de extracción como en las de reextracción, en los procesos hidrometalúrgicos, es posible que una etapa con circulación cruzada como la descrita se comporte de forma próxima a 1,5 etapas convencionales, y el número total de etapas necesarias se reduce en proporción que supera al $30 \%$, pudiéndose llegar al $50 \%$ en casos de reparto muy favorable.

En cada situación concreta tiene que calcularse las posibilidades de la etapa que opera en corriente

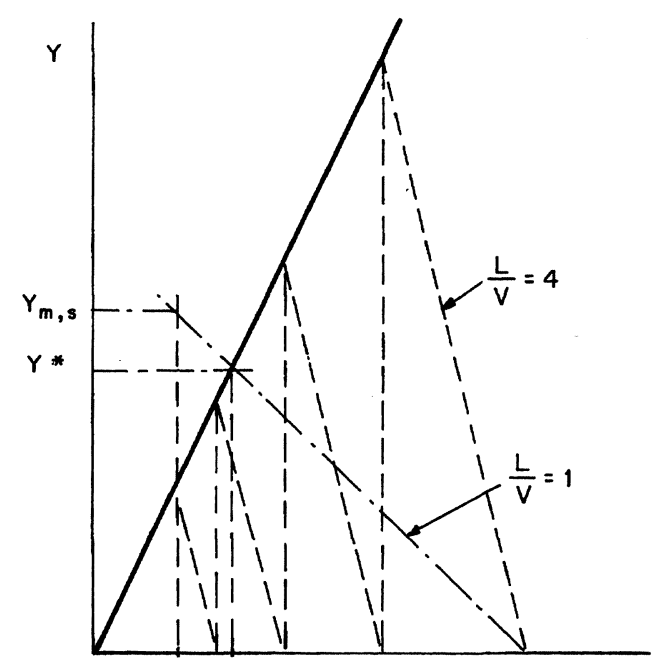

Fig. 2.- Evolución de $X$ e $Y$ para $L / V=1, N=1$ y $N L / V=4, N=4$.

FIG. 2.-Evolution of $\mathrm{X}$ versus $\mathrm{Y}$ for $\mathrm{L} / \mathrm{V}=1$, $\mathrm{N}=1$ and $\mathrm{NL} / \mathrm{V}=4, \mathrm{~N}=4$. 
cruzada, teniendo en cuenta las condiciones que puede imponer el diagrama de flujo del proceso hidrometalúrgico. A continuación se estudia, como ejemplo, la recuperación de uranio de soluciones fértiles procedentes del tratamiento ácido de minerales de uranio, mediante Alamine 366, 0,07M en queroseno, con un $3 \%$ de alcohol decílico. Se utilizan los datos de equilibrio presentados en (9), convenientemente correlacionados. Los datos de equilibrio utilizados no son lo suficientemente numerosos para que a la correlación que se presenta más adelante, se le pueda asignar una determinada cota de error. No obstante, para realizar los cálculos que conducen a los resultados que se estudian a continuación, la correlación da una base adecuada para su comparación.

Si se expresan las concentraciones $X$ e $Y$ en fase acuosa y orgánica respectivamente, en $\mathrm{g}_{3} \mathrm{O}_{8} / 1$, los datos de equilibrio se pueden calcular mediante las expresiones siguientes:

Para el intervalo $X=0, X=0,160$

$Y=44,48 X-238,20 X^{2}+419,62 X^{3}$

Para $X>0,160$

$Y=0,274$

En la tabla I se representan los datos de las composiciones de fases para $N=1,(L / V)=10$, correspondientes a cinco etapas teóricas. Las composiciones de entrada en el conjunto de las cinco etapas son, $X_{\mathrm{e}}=$ $0,273, Y_{\mathrm{e}}=0$, y las de salida, $X_{\mathrm{s}}=0,0004, Y_{\mathrm{s}}=2,73$.

Como ejemplo de utilización del algoritmo de cálculo expuesto anteriormente, en la tabla II se presentan también los datos de las composiciones de fases para $N=3,(L / V)=10$, correspondientes a tres etapas con circulación de fase orgánica segregada en tres corrientes. Como base de cálculo se utilizan las composiciones de fases, referidas al conjunto de tres etapas, $X_{\mathrm{s}}=0,0004, Y_{\mathrm{e}}=0$.

En la figura 3 se presentan los valores de $X_{\mathrm{s}}$ en todas las etapas, $\sin (N=1)$ y con circulación de fase orgánica segregada $(N=2$ hasta $N=6)$, con la misma base $X_{\mathrm{s}}=0,0004, Y_{\mathrm{e}}=0$ utilizada en el ejemplo de la tabla II. En ella puede comprobarse que con $N=4$, prácticamente se consiguen con tres etapas, los mismos valores de $X_{\mathrm{e}}, Y_{\mathrm{s}}$ correspondientes al conjunto de cinco etapas de contacto convencionales (Tabla I). Los valores de $X_{\mathrm{e}} Y_{\mathrm{s}}$ iguales se alcanzan con $N$ igual a cinco o seis.

\section{COMENTARIOS}

La variante de etapa de contacto líquido-líquido descrita, presenta en principio dos aspectos de interés en relación con los sistemas mezclador-sedimentador convencionales: a) su menor consumo de energía, y b) la mayor variación de concentraciones conseguida en cada etapa, con lo que se reduce su número.
TABla I.- Composición de fases: $N=1 ;(L / V)=10$; $(L / V) N=10$

TABLE I.- Phases composition: $\mathrm{N}=1 ;(\mathrm{L} / \mathrm{V})=10$; $(\mathrm{L} / \mathrm{V})=10$

\begin{tabular}{|c|c|l|c|c|c|}
\hline$n$ & $N$ & $Y_{\mathrm{e}}$ & $X_{\mathrm{s}}^{*}$ & $Y_{\mathrm{s}}^{*}$ & $X_{\mathrm{e}}=X_{\mathrm{s}}-\frac{Y_{\mathrm{e}}-Y_{\mathrm{s}}}{(L / V) N}$ \\
\hline 1 & 1 & 0 & 0,00040 & 0,0177 & 0,00217 \\
2 & 1 & 0,0177 & 0,00217 & 0,0956 & 0,00996 \\
3 & 1 & 0,0956 & 0,00996 & 0,420 & 0,0424 \\
4 & 1 & 0,420 & 0,0424 & 1,48 & 0,148 \\
5 & 1 & 1,48 & 0,148 & 2,73 & 0,273 \\
\hline
\end{tabular}

TABLA II.- Composición de fases: $N=3 ;(L / V)=$ $10 ;(L / V) N=30$

TABLE II.- Phases composition: $\mathrm{N}=3 ;(\mathrm{L} / \mathrm{V})=10$; $(\mathrm{L} / \mathrm{V}) \mathrm{N}=30$

\begin{tabular}{|c|c|c|c|c|c|}
\hline$n$ & $N$ & $Y_{\mathrm{e}}$ & $X_{\mathrm{s}}^{*}$ & $Y_{\mathrm{s}}^{*}$ & $X_{\mathrm{e}}=X_{\mathrm{s}}-\frac{Y_{\mathrm{e}}-Y_{\mathrm{s}}}{(L / V) N}$ \\
\hline 1 & 1 & 0 & 0,000400 & 0,0177 & 0,000992 \\
\hline 1 & 2 & 0 & 0,000992 & 0,0439 & 0,00245 \\
\hline 1 & 3 & 0 & 0,00245 & 0,1077 & 0,00604 \\
\hline 1 & & & $Y_{\mathrm{m}, \mathrm{s}}$ & 0,0564 & \\
\hline 2 & 1 & 0,0564 & 0,00604 & 0,260 & 0,0128 \\
\hline 2 & 2 & 0,0564 & 0,0128 & 0,533 & 0,0287 \\
\hline 2 & 3 & 0,0564 & 0,0287 & 1,091 & 0,0615 \\
\hline 2 & & & $Y_{\mathrm{m}, \mathrm{s}}$ & 0,611 & \\
\hline 3 & 1 & 0,611 & 0,0615 & 1,933 & 0,105 \\
\hline 3 & 2 & 0,611 & 0,105 & 2,535 & 0,170 \\
\hline 3 & 3 & 0,611 & 0,170 & 2,740 & 0,240 \\
\hline 3 & & & $Y_{\mathrm{m}, \mathrm{s}}$ & 2,40 & \\
\hline
\end{tabular}

En cada caso concreto, se debe evaluar esta reducción del número de etapas, ya que depende del número de fracciones de fase orgánica con las que se opere, así como de las características del diagrama de equilibrio del sistema considerado.

También se requiere el conocimiento experimental del comportamiento de fases acuosa y orgánica en el flujo ascendente, en corrientes paralelas, sobre un relleno convencional, con el fin de definir la altura de relleno necesaria, los caudales másicos aceptables y las condiciones en las que tiene lugar la coalescencia de la fase orgánica, para evaluar el espesor de la capa orgánica continua con la que se debe operar. Este aspecto es de gran importancia por la influencia que su elección correcta tiene al reducir el retenido de fase orgánica en operación y, por tanto, la reducción de la inversión necesaria, lo 


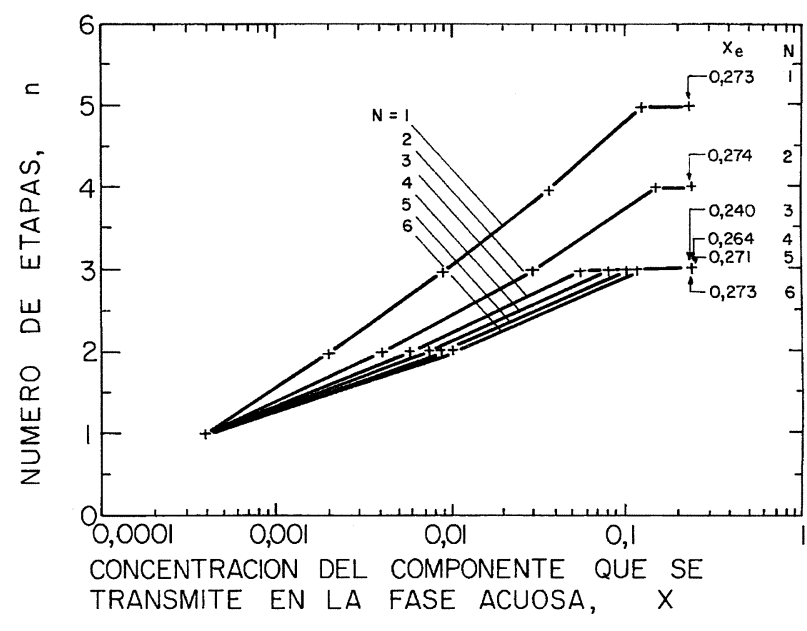

Fig. 3.- Evolución de $X$ en función de $n$ y $N$.

\section{FIG. 3.-Evolution of $\mathrm{X}$ versus $\mathrm{n}$ and $\mathrm{N}$.}

que constituye otra de las posibles ventajas de la nueva variante de etapa de contacto.

Es evidente que sin experimentación previa, no se puede realizar la evaluación de las pérdidas de fase orgánica en operación con la circulación de fases descrita. Sin embargo, cabe esperar que las pérdidas serán menores que las que se tienen en las etapas convencionales con mezcla de fases mediante agitación mecánica, ya que en los orificios que distribuyen la fase orgánica, en la base de los conductos verticales ascendentes, es probable que se produzca un menor número de pequeñas gotas de fase orgánica en relación con la agitación mecánica. Estas pequeñas gotas, como se indicó anteriormente, difícilmente coalescen y fácilmente son arrastradas por la fase acuosa y con frecuencia son las causantes de las pérdidas de fase orgánica en operación.

En el diseño de la etapa son posibles perfeccionamientos, en la disposición de bombas, conductos de fraccionamiento de fase orgánica, distribución y forma de orificios para la obtención de gotas de fase orgánica, distribución de flujo mediante vertederos almenados, tanto en fase orgánica como en fase acuosa, en este último caso diseñándolos con posibilidad de desplazamiento, manteniendo la línea base de rebose horizontal. Como complemento es conveniente disponer de una medida continua de posición de interfase orgánica-acuosa.

$\mathrm{La}$ introducción del relleno en los conductos ascendentes exige disponer, en las tapas, los convenientes agujeros de hombre con tapa fija o móvil, sin o con cierre hidráulico. La disposición del relleno, en la etapa, se puede efectuar en la forma usual, llenándola previamente de agua, y su retirada para limpieza, mediante un sistema de succión unido a un depósito colector.

Por último, se resalta que también es posible utilizar los conductos, en los que la fase acuosa desciende, para realizar la transferencia de materia en contracorriente. Los caudales de inundación limitan su realización práctica al exigir una mayor sección transversal.

\section{Nomenclatura}

$L$ Caudal de fase acuosa. Aqueous phase flow.

$N$ Número de fracciones en que se segrega la fase orgánica.

Number of splitted flow of the organic phase.

$V$ Caudal de fase orgánica. Organic phase flow.

$n$ Número de etapas. Number of stages.

$X$ Concentración del componente que se transmite en la fase acuosa.

Transmitted component concentration in the aqueous phase.

$Y$ Concentración del componente que se transmite en la fase orgánica.

Transmitted component concentration in the organic phase.

\section{Exponentes}

* Concentración en equilibrio. Equilibrium concentration.

\section{Subíndices}

e Entrada a la etapa.

Stage feed.

$\mathrm{m}$ Valor medio.

Mean value.

s Salida de la etapa.

Stage exit.

\section{REFERENCIAS}

1) Ritcey, G.M. y AshbrooK, A.W. Solvent extraction. Principles and applications to process metallurgy. Part II. pág. 49-71. Elsevier Scientific Publishing Co. AmsterdamOxford-Nueva York, 1979.

2) Power-Gas Limited, Power-Gas House, 8 Baker St., Londres, Reino Unido.

3) Denver Equipment Co., 1400 17th., Denver, CO, 80217, EE.UU.

4) IMI (Tami) Institute for Research and Development, P.O. Box 313, Haifa, Israel.

5) Lurgi Mineraloltechnik GmbH, 6000 Frankfurt (Main) 2, Lurgihans, Gervinusstrasse 17/19.

6) Holmes and Narver, Inc., 400 East Orangethorpe Ave., Anaheim, CA, 92801, EE.UU.

7) OTERo DE LA GÁNDARA, J.L. Aspectos teóricos de la transferencia de materia o calor en contracorriente cruzada. $A n$. Quim. 1993: 333-339.

8) OTERo DE LA GÁNDARA, J.L. Patente de invención española. № de solicitud 9202267 (11 de noviembre de 1992).

9) RegiFe, J.M. y CORDERo, G. Energía Nuclear, 9, 1965: 410. 